\title{
Prevalencia de adenomiomas y hallazgos asociados en estudios de resonancia magnética de útero en pacientes con miomatosis
}

\author{
Prevalence of adenomyomas and associated findings in magnetic \\ resonance studies of the uterus in patients with myomatosis
}

\author{
Lilian Nataly Galicia Téllez,* María Guadalupe Gómez Pérez ${ }^{\ddagger}$
}

Citar como: Galicia TLN, Gómez PMG. Prevalencia de adenomiomas y hallazgos asociados en estudios de resonancia magnética de útero en pacientes con miomatosis. Acta Med GA. 2022; 20 (1): 24-29. https://dx.doi.org/10.35366/103552

\section{Resumen}

Objetivos: Determinar características de la adenomiosis, la incidencia y establecer su asociación con otros hallazgos de imagen en mujeres sometidas a un estudio de resonancia magnética por miomatosis uterina. Material y métodos: Estudio tipo observacional, descriptivo, retrospectivo y longitudinal. Se revisaron 50 estudios de resonancia magnética con protocolo para localización de miomas realizados del $1 .^{\circ}$ de enero del 2018 al 31 de diciembre del 2019. Resultados: El promedio de edad fue de $38.72 \pm 9.12$ años, con distribución normal o paramétrica según la prueba de Kolmogórov-Smirnov ( $p=$ 0.2). En cuanto a la localización de las lesiones, predominó la intramural (30\%) y la asociación intramural-subserosa (22\%). El tamaño promedio de las lesiones fue de $9.76 \pm 4.44 \mathrm{~mm}$. De los 50 casos con diagnóstico clínico, sólo 11 (22\%) fueron compatibles con adenomiosis, por lo que se tuvieron 39 falsos positivos. La presencia de microquistes se observó en 15 pacientes (30\%). No se encontró correlación entre el tamaño de la lesión y su localización $(p=0.193)$. Conclusión: La resonancia magnética es un instrumento útil para discernir las lesiones de adenomiomatosis verdaderas de las que fueron diagnosticadas clínicamente.

Palabras clave: Adenomiosis, resonancia magnética, miomatosis.

\section{Abstract}

Objectives: To establish characteristics of adenomyosis and its incidence, as well as to establish the link between adenomyosis with other imaging findings of women undergoing an MRI study by uterine myomatosis. Material and methods: The following study has an observational, descriptive, retrospective, and longitudinal. $50 \mathrm{MRI}$ studies with the protocol for the location of fibroids were reviewed. Such studies were conducted between January 01, 2018, to December 31, 2019. Results: The average age was $38.72 \pm 9.12$ years with normal or parametric distribution according to the Kolmogórov-Smirnov test $(p=0.2)$. The lesson's location was predominantly in the intramural (30\%) and the intramural-subserous association (22\%). The average injury size was $9.76 \pm 4.44 \mathrm{~mm}$. Of the 50 cases with a clinical diagnosis, only 11 (22\%) were compatible with adenomyosis, for which there were 39 false positives. Microcysts were found in 15 patients $(30 \%)$. No correlation was found between the size of the injury and its location $(p=0.193)$. Conclusion: MRI is a useful tool for discerning real adenomyomatosis injuries from those diagnosed by clinical diagnosis.

Keywords: Adenomyosis, magnetic resonance, myomatosis.
* Residente de Alta Especialidad de Resonancia Magnética de Cuerpo Completo. Facultad Mexicana de Medicina de la Universidad La Salle. México.

₹ Profesora Titular del Curso de Alta Especialidad de Resonancia Magnética de Cuerpo Completo del Servicio de Resonancia Magnética.

Hospital Angeles Pedregal. México.
Correspondencia:

Lilian Nataly Galicia Téllez

Correo electrónico: galez25@outlook.es

Aceptado: 24-05-2021.

www.medigraphic.com/actamedica

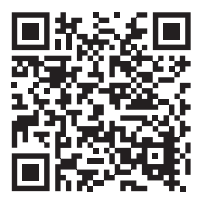




\section{INTRODUCCIÓN}

La adenomiosis es una enfermedad que afecta exclusivamente al tejido endometrial uterino sin que se involucre el miometrio. Fue descrita por primera vez en 1860 por el patólogo alemán Carl von Rokitansky y denominada en un inicio como cystosarcoma adenoids uterinum.

No existe explicación etiológica precisa de la adenomiosis. Se tiene como postulado más aceptado el hecho de que la disrupción en la continuidad entre las capas más profundas del endometrio con el miometrio da paso a lesiones que posteriormente se identificaron como adenomiomas. Dichas lesiones se ven inducidas por una proliferación inapropiada del endometrio, con el desarrollo de hipertrofia, hiperplasia y angiogénesis. ${ }^{2}$

Los datos epidemiológicos sobre adenomiosis no se consideran muy claros, debido a la forma en que se realiza el diagnóstico, se encuentra infradiagnosticado. Su rango de incidencia se tenía contemplado entre 5 y $7 \%$; pero actualmente se estima que puede alcanzar 20 a $35 \%{ }^{3}$ aunque hay series que señalan que la incidencia de la adenomiosis es de hasta $54 \%$ en estudios post mortem. En México, los datos señalan una incidencia de $26.2 \%$ o más, dependiendo de la población de estudio (Figura 1). ${ }^{4}$

La adenomiosis es clínicamente muy parecida a otras lesiones uterinas como los miomas, lo que dificulta su diagnóstico clínico. Dentro del cuadro clínico descrito se encuentran aumentos en la contracción uterina, sangrado

Figura 1: Incidencia de adenomiosis en la población de mujeres del ISSEMyM (Instituto de Seguridad Social del Estado de México y Municipios) Satélite entre 2007 y 2010.

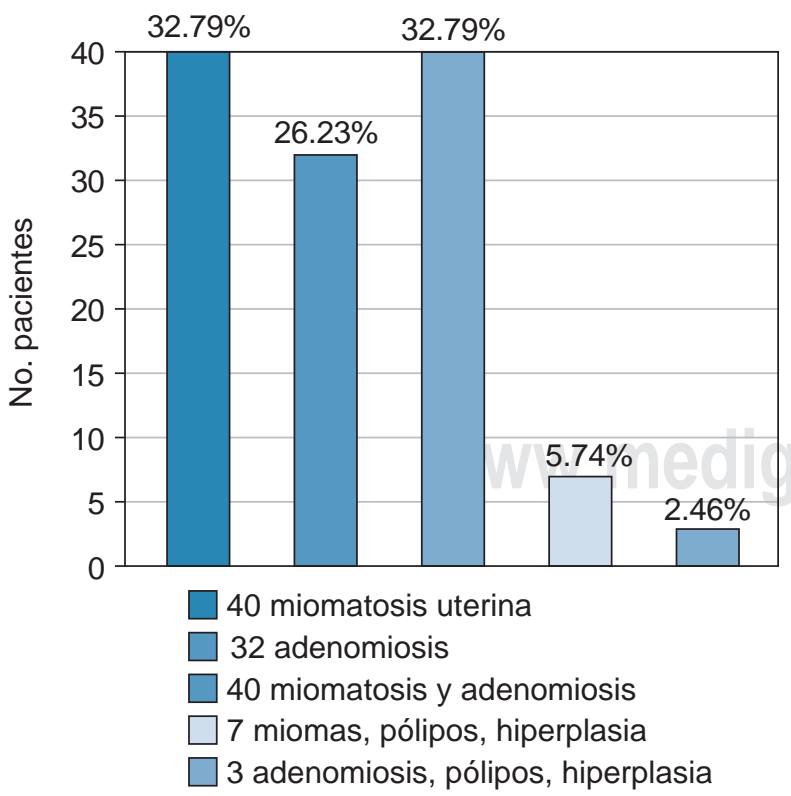

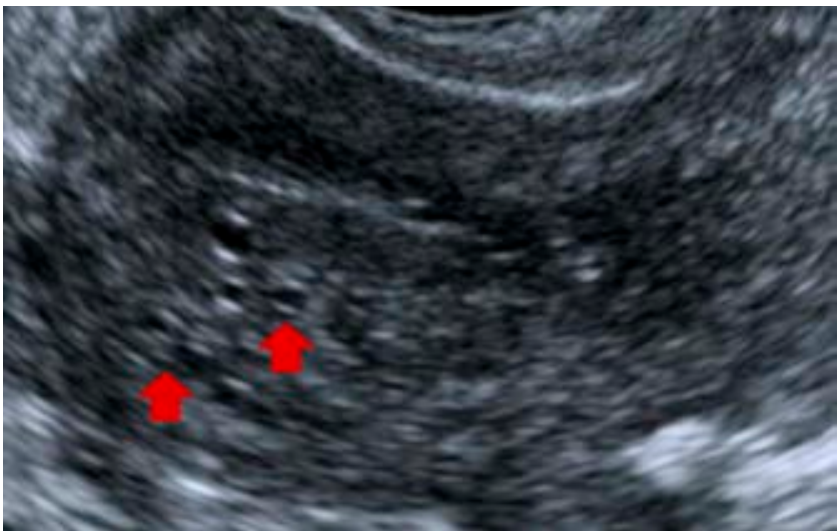

Figura 2: Ultrasonido endovaginal corte sagital que muestra imágenes quísticas y anecoicas de tamaño variable distribuidas en el miometrio.

y dismenorrea. ${ }^{5}$ También se ha descrito dolor pélvico y dispareunia asociada directamente con la adenomiosis; aunque hasta $33 \%$ de los casos de adenomiosis se considera asintomático. ${ }^{5}$

Los estudios de laboratorio resultan inútiles, ya que no existe un marcador específico para ello. Por lo que los métodos empleados y recomendados para el diagnóstico son los de imagen, en especial la resonancia magnética, que tiene una sensibilidad de $89 \%$ y una especificidad de $86 \%{ }^{6}{ }^{6}$ Pero debido a los costos, se recomienda el uso de ultrasonido, mientras que la resonancia sólo se reserva para los casos de difícil diagnóstico o en los que los diagnósticos previos se han considerado erróneos.

Los adenomiomas se forman de estroma endometrial y tejido glandular que desplazan al músculo del miometrio; típicamente, dichas lesiones son descritas con una profundidad de 2.5-8 mm en el miometrio, aunque se han hecho señalamientos de que la medición debe considerarse en relación con el porcentaje de invasión en el miometrio. ${ }^{3}$

Para el uso de ultrasonido se recomienda un abordaje transvaginal, encontrando infiltración endometrial: con estriaciones ecogénicas y nódulos, quistes miometriales y divertículos "lollipop"; así como proliferación de músculo suave, ya sea de tipo focal o difuso con bordes en el miometrio. En cuanto a la vascularidad, mediante ultrasonido Doppler se observa aumento de la angiogénesis con vasos tortuosos. La mayor sensibilidad en el diagnóstico se logra con el uso de la resonancia magnética, en la que los haIlazgos son similares a los observados en el ultrasonido; ${ }^{8}$ dentro de estas observaciones se entiende lo siguiente: ${ }^{9}$ en las imágenes T2 se observa alargamiento uterino con regiones más definidas y de baja intensidad con hiperplasia del músculo liso (zona de unión de más de $12 \mathrm{~mm}$ ), quistes miometriales hiperintensos que reflejan regiones 
del tejido miometrial ectópico; con posible señal T1 intrínseca incrementada o con mayor sensibilidad para focos hemorrágicos. No existe recomendación de mejora con el medio de contraste, ya que no permite valorar o identificar la vascularización de la zona (Figura 2).

Adicionalmente, los hallazgos por resonancia magnética pueden ser clasificados como signos directos o indirectos. Dentro de los directos se encuentran los microquistes, siendo éstos el principal signo de adenomiosis identificable por resonancia magnética, los cuales tienen un tamaño de entre $2-7 \mathrm{~mm}$, con una media de $3 \mathrm{~mm}$ y se encuentran dentro del miometrio (Figura 3), ${ }^{10}$ además de que se hallan principalmente en la zona de unión y raramente en el miometrio; son hipointensas en T1 e hiperintensas en T2, e identificables de mejor manera al final del periodo menstrual con cavidad quística. ${ }^{11}$ Los adenomiomas que se encuentran como una consolidación focal de las glándulas adenomióticas dentro del miometrio se manifiestan como una masa de paredes bien delimitadas en la zona de unión, por lo que el principal diagnóstico diferencial es el leiomioma uterino, ya que ambas presentan el mismo grado de intensidad, con la única diferencia que la vascularidad de los adenomiomas presenta más angiogénesis (Figura 4). ${ }^{12}$

Por otro lado, los signos indirectos corresponden a hallazgos como el espesor de la zona de unión que, después de los microquistes, son el signo más estudiado en

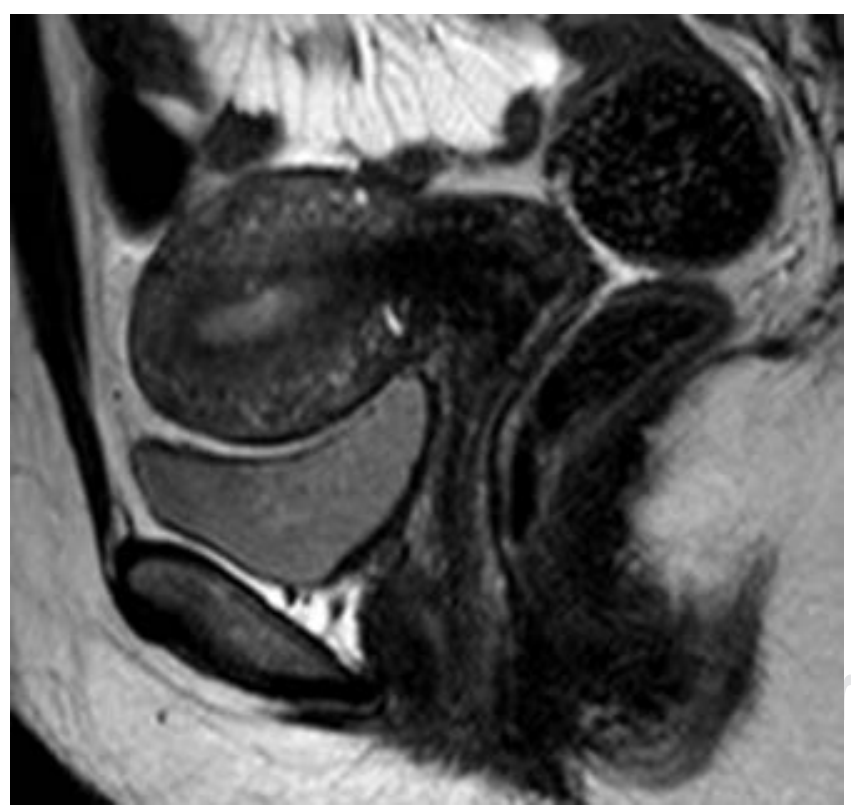

Figura 3: Secuencia de útero en plano sagital donde se observa patrón heterogéneo secundario a múltiples trayectos de aspecto tubular de comportamiento hiperintenso por dilataciones quísticas de las glándulas endometriales relacionadas con adenomiosis.

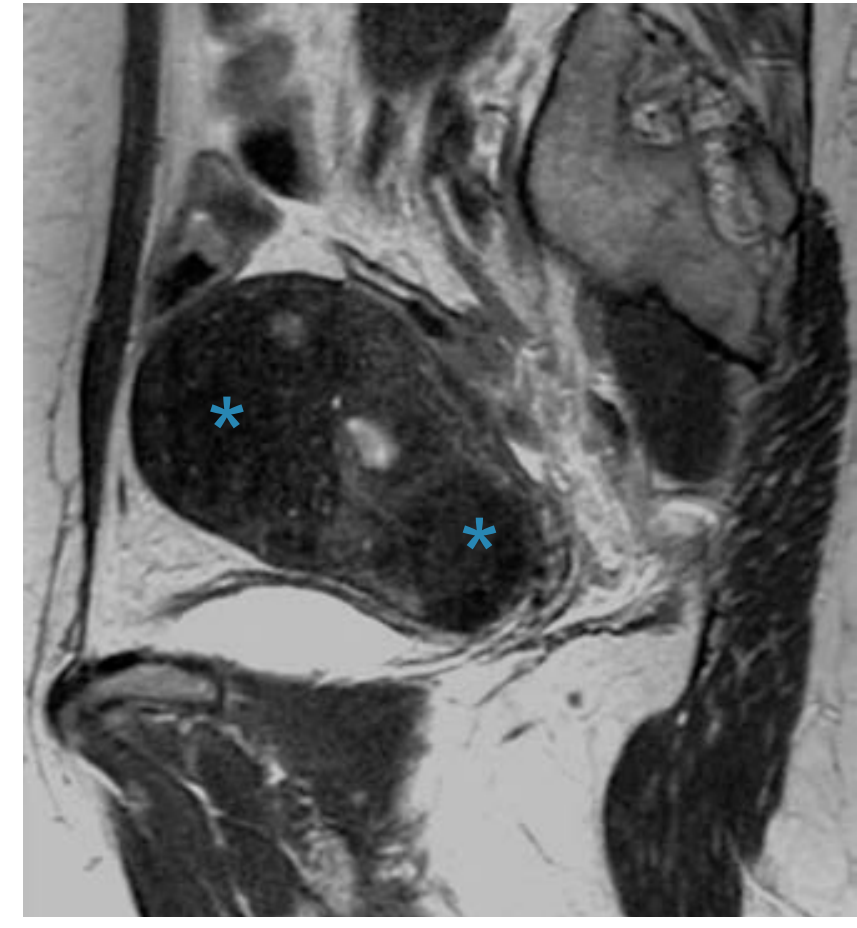

Figura 4: Imagen sagital T2 de alta resolución en paciente con adenomiosis. Útero aumentado de tamaño en el que se visualizan zonas de adenomiosis focal (*) marcadamente hipointensas respecto al parénquima normal que queda entre ambas. En el espesor del miometrio engrosado en fundus, visibles varios focos hiperintensos que representan degeneración quística y glándulas ectópicas.

imagen para la adenomiosis. Esta zona suele engrosarse en los casos de adenomiosis, e incluso se pueden presentar microquistes en dicho engrosamiento. El valor medio del grosor de la zona de unión es de $12 \mathrm{~mm}$; un aumento por arriba de ese grosor es indicativo de adenomiosis con $85 \%$ de certeza y $96 \%$ de especificidad. ${ }^{13}$ Otro dato indirecto corresponde a la zona de unión diferencial con un concepto introducido en 2001 por Dueholm; esta zona está calculada como la diferencia entre el espesor más delgado o grueso en la parte anterior y posterior del útero, la cual debe ser menor de $5 \mathrm{~mm}$ para ser considerada regular; $;^{14}$ aunque cabe señalar que si la proporción es mayor a $40 \%$, en caso de miomatosis coexistente, no se considera un valor diagnóstico útil. ${ }^{15}$

Debido a los posibles falsos positivos obtenidos como resultado de la fisiología uterina, se recomienda realizar más de dos tomas de imagen de la zona de unión en T2 en diferentes momentos del estudio, o bien, el uso de un inhibidor de la contracción uterina previo al inicio de la resonancia. ${ }^{16}$ Por ello, es recomendable que el estudio de resonancia se realice en el día proliferativo o secretorio 
para disminuir la influencia de la función fisiológica del útero. ${ }^{8}$ Además, la sensibilidad y especificidad de la resonancia magnética se ha reportado similar e incluso mejor en comparación con el ultrasonido. ${ }^{9}$

Como se comentó previamente, algunos de los indicadores empleados para la valoración por imagen de la adenomiosis no son útiles en presencia de otra patología uterina; lo cual resulta ser más común de lo pensado, ya que estudios en México han señalado que hasta $61.35 \%$ de las pacientes con adenomiomas presentan una enfermedad añadida, como la miomatosis uterina (Figura 5); lo cual implica que ambas patologías tienden a coexistir. ${ }^{4}$ Sin embargo, en muchos casos, el diagnóstico diferencial entre la miomatosis uterina y la adenomiomatosis se da en el periodo postquirúrgico, cuando las piezas obtenidas de biopsia o histerectomía son analizadas. ${ }^{17,18}$

En las últimas décadas, la resonancia magnética se ha convertido en una de las técnicas de imagen y de diagnóstico más socorridas para distintas especialidades, por lo que es necesario aprovechar su uso para mejorar el diagnóstico de diversas enfermedades.

En muchas ocasiones, el uso de herramientas de diagnóstico por imagen también permite identificar, de manera no premeditada, otro tipo de lesiones, o incluso realizar hallazgos diagnósticos diferentes a los planteados al inicio del abordaje clínico; entre éstos, están los que se dan a nivel ginecológico, como la adenomiosis, la cual puede encontrarse o asociarse en el estudio de los miomas. Sin embargo, es necesario identificar la frecuencia y las características que se presentan de manera simultánea en ambos diagnósticos, con la intención de optimizar la resonancia diagnóstica para su uso. ${ }^{4}$

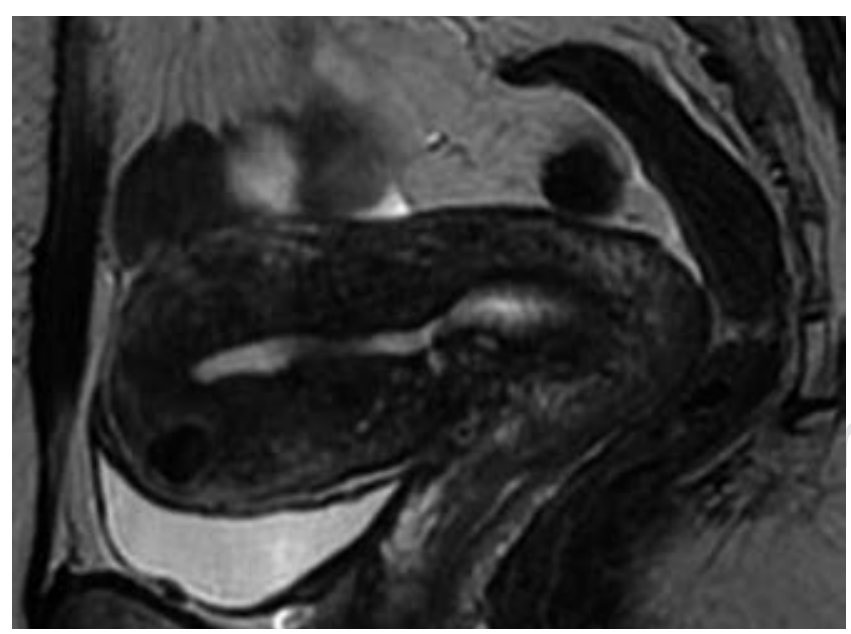

Figura 5: Imagen sagital T2 de alta resolución con imágenes en relación a adenomiosis difusa y pequeño mioma intramural en la pared anterior.
Tabla 1: Distribución por localización.

\begin{tabular}{lrr} 
& $n$ & $\%$ \\
\hline $\begin{array}{lr}\text { Sin adenomiosis } \\
\text { Intramurales }\end{array}$ & 6 & 12 \\
Intramurales y & 15 & 30 \\
submucosos & 6 & 12 \\
Intramurales y & 11 & 22 \\
subserosos & & \\
Intramural, & 4 & 8 \\
submucoso y & & \\
subseroso & & \\
Submucoso & 3 & 10 \\
Subseroso & 5 &
\end{tabular}

Con base en lo planteado, se realizó este estudio con el objetivo de determinar las características e incidencia de la adenomiosis, y establecer su asociación con otros hallazgos de imagen en mujeres sometidas a un estudio de resonancia magnética por miomatosis uterina.

\section{MATERIAL Y MÉTODOS}

Estudio tipo observacional, descriptivo, retrospectivo y longitudinal en el que se revisaron 50 estudios de resonancia magnética con protocolo para localización de miomas realizados del $1^{\circ}$ de enero del 2018 al 31 de diciembre del 2019.

Las pacientes fueron recibidas en el Departamento de Resonancia Magnética del Hospital Angeles Pedregal con solicitud para la realización de protocolo para localización de miomas y con hoja de consentimiento informado.

Los estudios fueron realizados con equipo General Electric Signa de 1.5 Tesla con protocolo para estudio de localización de miomas con secuencias potenciadas en T2 en los planos coronal, sagital y axial, así como axial T2 FINOS PROPELLER, coronal T2 PROPELLER y sagital T2 FINOS PROPELLER.

Se analizaron los estudios en el Sistema de Comunicaciones y Archivado de imágenes (PACS) Carestream así como los reportes hechos por el médico radiólogo experto en resonancia magnética y por el médico residente de alta especialidad de cuerpo completo.

Posteriormente, se creó una base de datos para el análisis estadístico, el cual se llevó a cabo mediante el programa IBM SPSS v26 (USA).

\section{RESULTADOS}

El promedio de edad de la población en estudio fue de $38.72 \pm 9.12$ años de edad, con una distribución normal o 


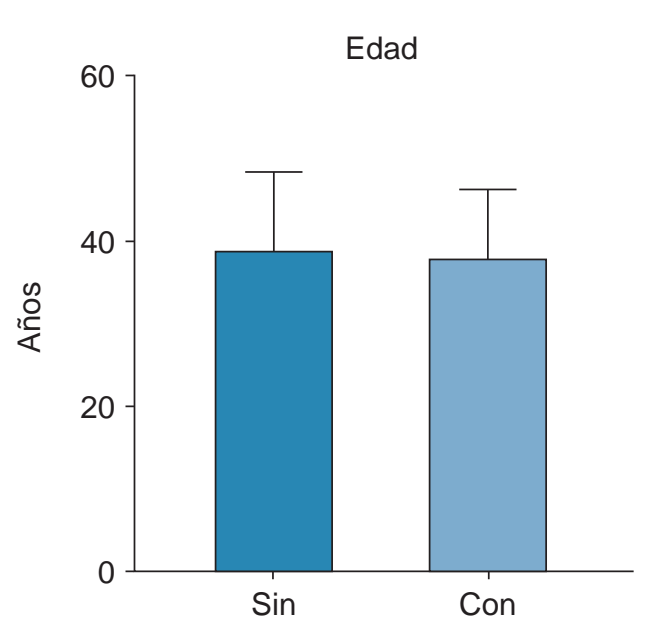

Figura 6:

Promedio de edad y tamaño de las lesiones. El promedio de edad de la población en estudio fue de 38.72 \pm 9.12 años de edad. El tamaño promedio de las lesiones encontradas fue de $9.76 \pm 4.44 \mathrm{~mm}$. * $p \leq 0.05$. paramétrica según la prueba de Kolmogórov-Smirnov $(\mathrm{p}=$ $0.2)$. En cuanto a la localización de las lesiones observadas mediante el estudio de resonancia magnética, se encontró la distribución mostrada en la Tabla 1. El tamaño promedio de las lesiones encontradas fue de $9.76 \pm 4.44 \mathrm{~mm}$ (Figura 6).

De los 50 casos con diagnóstico clínico, sólo 11 (22\%) fueron compatibles con adenomiosis, por lo que se tuvieron 39 falsos positivos.

La presencia de microquistes se encontró en 15 pacientes (30\%), el resto no tuvo datos de lesiones microquísticas.

Posteriormente, se realizó una separación de los casos en dos grupos: los que presentaron adenomiomas y los que no. Los datos observados se presentan en la Tabla 2. No se encontró correlación entre el tamaño de la lesión y su localización ( $p=0.193)$.

\section{DISCUSIÓN}

La adenomiosis o adenomiomatosis se caracteriza típicamente por ser diagnosticada en la quinta década de la vida; sin embargo, en nuestro estudio se identificó una edad diagnóstica menor que en otras series. ${ }^{19,20}$ Aunque estudios de cortes más amplios señalan que en una proporción inferior a 20\%, derivado de factores genéticos, fumar, toxemias y otros, la edad promedio en la que se detecta la adenomiosis es menor a los 40 años, como sucede en nuestro estudio. ${ }^{21}$

No se han encontrado reportes sobre el tamaño promedio de las lesiones identificadas mediante resonancia magnética. Sin embargo, se ha informado que la lesión mínima detectable es de $0.5 \mathrm{~mm}$, lo cual podría explicar por qué en algunos casos no se identificaron las lesiones. ${ }^{22}$

En cuanto a la certeza del diagnóstico y su especificidad, se ha señalado que los estudios de imagen superan la capacidad diagnóstica del clínico. El ultrasonido da una

\begin{tabular}{|c|c|c|c|}
\hline & $\begin{array}{c}\text { Sin } \\
\text { adenomiosis }\end{array}$ & $\begin{array}{c}\text { Con } \\
\text { adenomiosis }\end{array}$ & $\mathrm{p}$ \\
\hline Edad (años)* & $38.92 \pm 9.49$ & $38.09 \pm 8.16$ & 0.7949 \\
\hline $\begin{array}{l}\text { Tamaño de la lesión } \\
(\mathrm{mm})^{\star}\end{array}$ & $8.51 \pm 3.33$ & $13.4 \pm 5.37$ & $<0.001$ \\
\hline Lugar de lesión, n (\%) & & & 0.513 \\
\hline Intramurales & $11(28.2)$ & $4(36.36)$ & \\
\hline Submucoso & $3(7.69)$ & $0(0)$ & \\
\hline Subseroso & $5(12.82)$ & $0(0)$ & \\
\hline $\begin{array}{l}\text { Intramural y } \\
\text { submucoso }\end{array}$ & $5(12.82)$ & $1(9)$ & \\
\hline $\begin{array}{l}\text { Intramural y } \\
\text { subseroso }\end{array}$ & $7(17.94)$ & $4(36.36)$ & \\
\hline $\begin{array}{l}\text { Intramural, } \\
\text { submucoso y } \\
\text { subseroso }\end{array}$ & $4(10.25)$ & $0(0)$ & \\
\hline No identificado & $4(10.25)$ & $2(18.18)$ & \\
\hline Microquistes, n (\%) & & & 0.86 \\
\hline Sí & $12(30.7)$ & $3(27.27)$ & \\
\hline No & $27(69.2)$ & $8(72.72)$ & \\
\hline
\end{tabular}

sensibilidad de $63 \%$ y una especificidad de $97 \%$ respecto a los estudios de patología, valores que pueden ser superados por los estudios de resonancia magnética T2. ${ }^{21}$

El presente estudio es una base para el conocimiento epidemiológico de las adenomiosis en la población mexicana, la cual ha sido muy poco analizada. Debe ampliarse el estudio comparativo con ultrasonido y patología clínica para identificar la sensibilidad y demás características de la adenomiosis en la población mexicana. 


\section{CONCLUSIONES}

La resonancia magnética es un instrumento útil para discernir las lesiones de adenomiomatosis diagnosticadas clínicamente. Con la resonancia magnética es posible distinguir los falsos positivos establecidos mediante la clínica y, de esta manera, tener acceso a un diagnóstico oportuno no invasivo, como puede ser un estudio de patología.

Sería recomendable realizar estudios en los que se incluyeran resultados de patología clínica para poder establecer la sensibilidad y especificidad con respecto al estándar de oro diagnóstico.

\section{REFERENCIAS}

1. Benagiano G, Brosens I, Lippi D. The history of endometriosis. Gynecol Obstet Invest. 2014; 78 (1): 1-9.

2. Taran FA, Stewart EA, Brucker S. Adenomyosis: epidemiology, risk factors, clinical phenotype and surgical and interventional alternatives to hysterectomy. Geburtshilfe Frauenheilkd. 2013; 73 (9): 924-931.

3. Abbott JA. Adenomyosis and abnormal uterine bleeding (AUB-A)pathogenesis, diagnosis, and management. Best Pract Res Clin Obstet Gynaecol. 2017; 40: 68-81.

4. Contreras Zarza E, Urias Soto FA. Incidencia de adenomiosis vs miomatosis uterina como causa de histerectomía en el hospital de concentración ISSEMYM Satélite entre el 1 de marzo de 2007 y el 1 de marzo de 2010 [Tesis]. México: Universidad Autónoma del Estado de México; 2013. Disponible en: http://repositorioslatinoamericanos. uchile.cl/handle/2250/977253

5. Struble J, Reid S, Bedaiwy MA. Adenomyosis: a clinical review of a challenging gynecologic condition. J Minim Invasive Gynecol. 2016; 23 (2): 164-185.

6. Reinhold C, McCarthy S, Bret PM, Mehio A, Atri M, Zakarian R et al. Diffuse adenomyosis: comparison of endovaginal US and MR imaging with histopathologic correlation. Radiology. 1996; 199 (1): 151-158.

7. Cunningham RK, Horrow MM, Smith RJ, Springer J. Adenomyosis: a sonographic diagnosis. Radiographics. 2018; 38 (5): 1576-1589.

8. Takeuchi M, Matsuzaki K. Adenomyosis: usual and unusual imaging manifestations, pitfalls, and problem-solving MR imaging techniques. Radiographics. 2011; 31 (1): 99-115.
9. Novellas S, Chassang M, Delotte J, Toullalan O, Chevallier A, Bouaziz J et al. MRI characteristics of the uterine junctional zone: from normal to the diagnosis of adenomyosis. AJRAm J Roentgenol. 2011; 196 (5): 1206-1213.

10. Taourel P, Laffargue G, Dechaud H. Adenomyosis: what imaging modality in the diagnosis and staging? Gynecol Obstet Fertil. 2004; 32 (11): 976-980.

11. Tamai K, Koyama T, Umeoka S, Saga T, Fujii S, Togashi K. Spectrum of MR features in adenomyosis. Best Pract Res Clin Obstet Gynaecol. 2006; 20 (4): 583-602.

12. Siegler AM, Camilien L. Adenomyosis. J Reprod Med. 1994; 39 (11): 841-853.

13. Kissler S, Zangos S, Kohl J, Wiegratz I, Rody A, Gatje R et al. Duration of dysmenorrhoea and extent of adenomyosis visualised by magnetic resonance imaging. Eur J Obstet Gynecol Reprod Biol. 2008; 137 (2): 204-209.

14. Dueholm M, Lundorf E, Hansen ES, Sorensen JS, Ledertoug $\mathrm{S}$, Olesen F. Magnetic resonance imaging and transvaginal ultrasonography for the diagnosis of adenomyosis. Fertil Steril. 2001; 76 (3): 588-594.

15. Cammilli F, Rizzello F, Castellacci E, Riviello C, Coccia ME. OP11.08: Transvaginal ultrasound versus magnetic resonance in the study of under-diagnosed endometriosis: adenomyosis and deep endometriosis. Ultrasound Obstet Gynecol. 2007; 30 (4): 492.

16. Nakai A, Koyama T, Fujimoto K, Togashi K. Functional MR imaging of the uterus. Magn Reson Imaging Clin N Am. 2008; 16 (4): 673684 , ix.

17. Cruz GG. Concordancia clínica e histopatología de miomatosis uterina en el Servicio de Ginecología en un hospital de tercer nivel [Tesis de posgrado]. Veracruz: Hospital Regional de Alta Especialidad de Veracruz; 2012.

18. Arellano PEI, Labastida TJ. Prevalencia de adenomiosis en piezas quirúrgicas de histerectomía y factores de riesgo clínicos relacionados. Acta Med. 2018; 16 (1): 15-22.

19. Joshi JK, Kirk L. Adenomyomatosis. In: StatPearls. Treasure Island (FL): StatPearls Publishing; 2021. Available in: https:/www.statpearls.com/ articlelibrary/viewarticle/17202

20. Jiménez ES, Cortéz LJ. Adenomiosis uterina estudio clínico epidemiológico. Rev Peru Ginecol Obstet. 1989; 35 (7): 14-19.

21. Harada T, Khine YM, Kaponis A, Nikellis T, Decavalas G, Taniguchi F. The impact of adenomyosis on women's fertility. Obstet Gynecol Surv. 2016; 71 (9): 557-568.

22. Ascher SM, Jha RC, Reinhold C. Benign myometrial conditions: leiomyomas and adenomyosis. Top Magn Reson Imaging. 2003; 14 (4): 281-304. 